\title{
Malfunción valvular intermitente por migración del catéter peritoneal a escroto
}

\author{
Shunt dysfunction secondary to peritoneal catheter migration to the scrotum
}

\author{
Dr. Lic. Mónica Rivero-Garvía ${ }^{a}$ Lic. José Luis Barbeito Gaído ${ }^{a}$ Lic. Juan Morcillo y Dr. Lic. Javier Márquez Rivas
}

\begin{abstract}
RESUMEN
Introducción. La malfunción valvular por obstrucción del catéter proximal es un hecho relativamente frecuente en el servicio de urgencias de los grandes hospitales, pero la obstrucción distal de causa no infecciosa es excepcional.

Caso clínico. Presentamos un varón de 6 años con malfunción valvular aguda, por migración del catéter distal al hemiescroto derecho. Para resolver el cuadro completamente se realizó un cierre del conducto peritoneo-vaginal de urgencia.

Discusión. La permeabilidad del conducto peritoneo-vaginal (existente en hasta el 60\% de los varones menores de 1 año) es la causante de los hidroceles en los pacientes portadores de una derivación ventrículo-peritoneal. Pero la migración del catéter es muy infrecuente, sobre todo en mayores de un año. Si este hecho conlleva el aumento de la presión intracraneal con disfunción valvular, el cierre de urgencia de dicho conducto debe ser prioritario.

Palabras clave: malfunción valvular, migración de catéter, hidrocele, hidrocefalia.
\end{abstract}

\section{SUMMARY}

Introduction. Valvular dysfunction secondary to obstruction of proximal catheter is relatively frequent at emergency room. However non-infectius obstruction of distal catheter is exceptional.

Case report. A 6-year-old boy with dysfunction shunt due to migration of the abdominal catheter into the right scrotum. The patient was operated urgently for peritoneum-vaginal processus closure.

Discussion. Permeability of the peritoneum-vaginal processus (until $60 \%$ of boys younger than one year) causes hydrocele in patients with ventricle-peritoneal shunt. However migration of the peritoneal cateter is very infrequent specially in patients older than one year. In the case of this event with increased intracraneal pressure levels and shunt malfunction, emergency closure of the duct should be a priority.

Key words: disfuntion shunt, migration distal catether, hydrocele, hydrocephalus.

http:/ /dx.doi.org/10.5546/aap.2013.e14

a. Unidad de Neurocirugía Pediátrica.

Hospital Vírgen del Rocío. Sevilla, España.

Correspondencia:

Dr. Lic. Mónica Rivero-Garvía: monicargarvia@msn.com

Conflicto de intereses: Ninguno que declarar.

Recibido: 4-6-2012

Aceptado: 25-7-2012

\section{INTRODUCCIÓN}

Las disfunciones valvulares por hipertensión localizada en el extremo distal del catéter de derivación son bien conocidas. En la población pediátrica es muy frecuente en situaciones de estreñimiento pertinaz, seudoquistes abdominales o peritoneos hiporreabsortivos por cirugías abdominales reiteradas.

Presentamos el caso de un paciente, mayor de un año, con hipertensión intracraneal secundaria a obstrucción funcional valvular por migración a la bolsa escrotal del catéter distal.

\section{CASO CLÍNICO}

Varón de 6 años, portador de derivación ventrículo-peritoneal desde el primer año de vida por hidrocefalia arreabsortiva secundaria a encefalitis connatal.

Acude al Servicio de Urgencias por cefalea intensa, vómitos y disminución del nivel de conciencia.

En la TC craneal se observa respecto de los estudios previos, dilatación del sistema ventricular (Figura 1). En el estudio del trayecto valvular se observa que el catéter peritoneal ha migrado hasta el hemiescroto derecho (Figura 2).

Tras reexplorar al paciente se observa aumento de la bolsa escrotal derecha. Se reintroduce el catéter de forma manual en el abdomen, pero a las pocas horas se repite el cuadro con migración a la bolsa escrotal, por lo que se decide la intervención quirúrgica urgente para cierre del defecto de la pared abdominal. Después de la cirugía el paciente permanece asintomático.

\section{DISCUSIÓN}

En la bibliografía se describen numerosas causas de disfunción distal: estreñimiento pertinaz, seudoquistes abdominales o peritoneos hiporreabsortivos por cirugías abdominales reitearadas, y numerosas alteraciones escrotales secundarias a catéter distal de las derivaciones ventrículo-peritoneales: migración, exposición, heridas por decúbito. La aparición de hidrocele en niños con derivación ventrículo-peritoneal no es infrecuente, tampoco la migración a escro- 
to, como se ha descrito en numerosos artículos. ${ }^{1-4}$ Sin embargo, el malfuncionamiento intermitente por migración a la bolsa escrotal es excepcional.

En la edad infantil, la etiopatogenia de las hernias inguinales indirectas es secundaria a la permeabilidad del conducto peritoneo-vaginal (cavidad por donde descienden los testículos desde el abdomen a partir de la $28^{\text {ava }}$ semana de gestación). Este conducto es permeable en el $60 \%$ de los niños menores de un año, hasta en el $40 \%$ de los mayores de un año y en casi un $20 \%$ de la población adulta, la mayoría de las veces de forma asintomática. Además, es más frecuente su persistencia en varones y en el lado derecho, como en nuestro caso. Las migraciones a escroto son más frecuentes durante el primer año de vida; ${ }^{3}$ por ello, este caso es realmente excepcional, pues el paciente tiene 6 años de edad.

Esta comunicación entre el peritoneo y la bolsa escrotal permite la migración de los dispositivos intraperitoneales o de cualquier clase de líquido abdominal. Además, la localización escrotal del catéter peritoneal es motivo también de lesión por decúbito con exposición del catéter a nivel escrotal, escrotos agudos por torsión o hidroceles masivos. ${ }^{4,5}$

FIGURA 1. TC de cráneo (corte axial) importante dilatación de las astas temporales y la cavidad poroencefálica frontal derecha, como signos de hidrocefalia aguda.

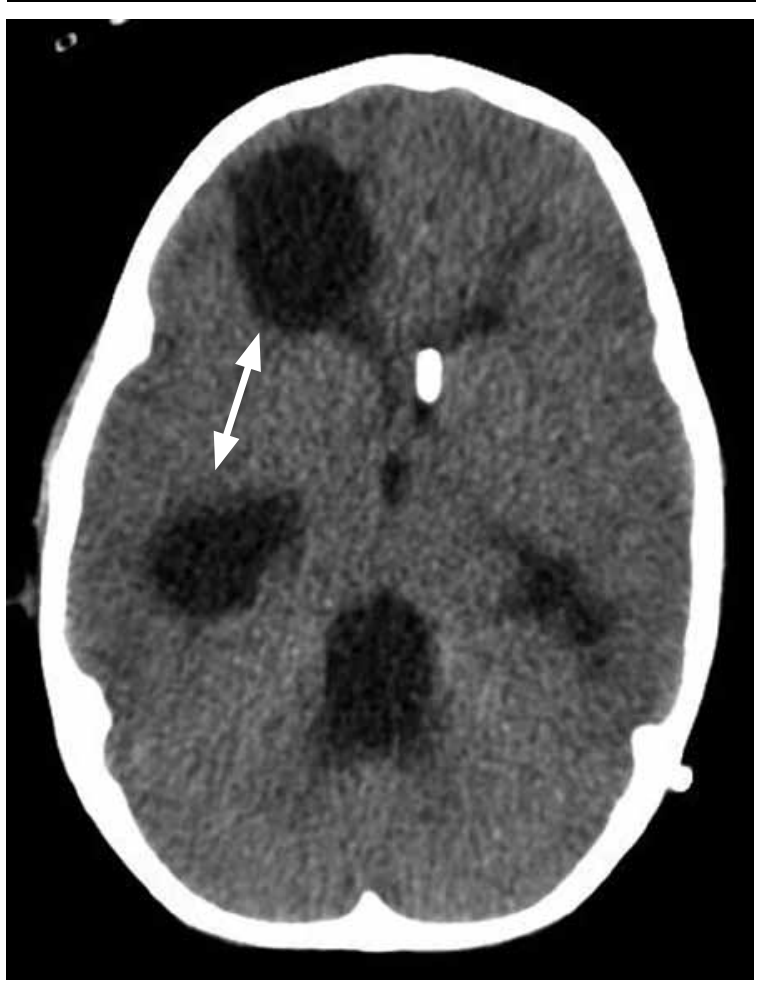

La migración a escroto no es excepcional, pero sí lo es que sea la causa de una malfunción valvular. En la bibliografía sólo existen dos casos de malfunción del dispositivo por dicho mecanismo: ${ }^{1-4}$ el acúmulo de líquido en la bolsa escrotal,

FIGURA 2. Radiografías simples del trayecto valvular. A: catéter distal intraperitoneal en el control postquirúrgico. B: catéter distal en escroto derecho, realizada en el Servicio de Urgencias.
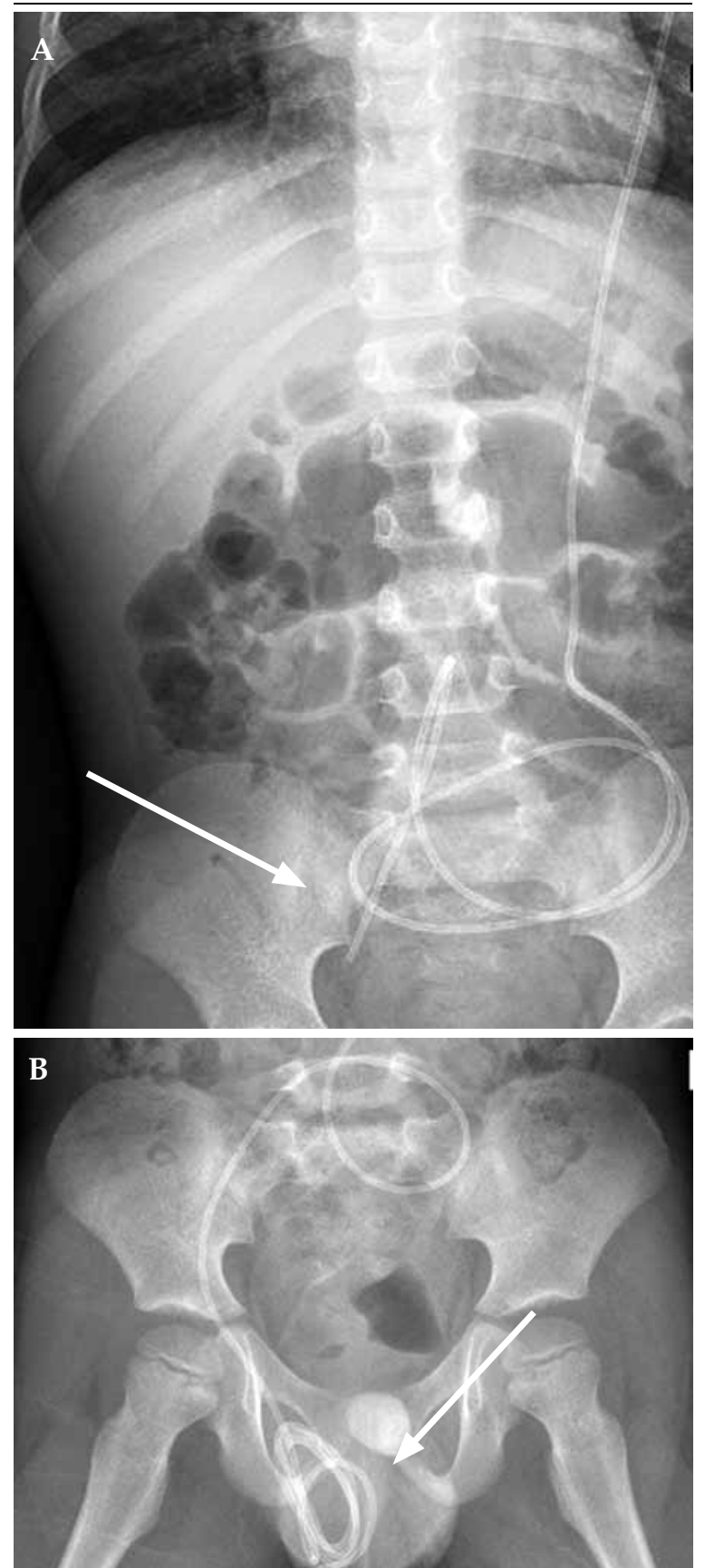
con un mecanismo de tipo compuerta, que impide su retorno a la cavidad peritoneal, provoca hipertensión localizada y disfunción valvular consecuente pues, al encontrarse el extremo distal del catéter peritoneal en una cavidad con presión mayor a la intracraneal, no se produce la salida de LCR y aumenta la presión intracraneal, ocasionando entonces un cuadro de hidrocefalia aguda.

El primer tratamiento realizado fue la reintroducción manual del catéter en la cavidad abdominal, en el propio servicio de Urgencias. A las pocas horas, volvieron a presentarse los mismos síntomas con nueva migración a la bolsa escrotal, por lo que se decidió, de forma conjunta con el equipo de cirugía pediátrica, la intervención urgente para reintroducir el catéter en la cavidad abdominal a través de una incisión inguinal, con cierre definitivo del conducto peritoneo-vaginal.

El tratamiento de las complicaciones escrotales (hidroceles, migración) después del primer año de vida es quirúrgico (cierre del conducto peritoneo- vaginal), pues la posibilidad de cierre espontáneo es muy baja. Si estas alteraciones aparecen antes del primer año de vida puede intentarse un manejo conservador, pues la mitad de los conductos se cierran de forma espontánea.

\section{BIBLIOGRAFÍA}

1. Villarejo FJ, Blázquez MG. Malfunctioning ventriculoperitoneal shunt due to extrusion of the abdominal catheter into the scrotum. J Neurosurg Sci 1980;24(3-4):187-9.

2. RamZ,FindlerG, GuttmanI, CherniakR, etal. Ventriculoperitoneal shunt malfunction due to migration of the abdominal catheter into the scrotum. J Pediatr Surg 1987;22(11):1045-6.

3. Fuwa I, Matsukado Y, Itoyama Y, Yokota A. Migration of a dissected peritoneal shunt catheter into the scrotum. Brain Dev 1984;6(3):336-8.

4. Albala DM,DanaherJW,HuntsmanWT. Ventriculoperitoneal shunt migration into the scrotum. Am Surg 1989;55(11):685-8.

5. De Aquino HB, Carelli EF, Borges Neto AG, Pereira CU. Nonfunctional abdominal complications of the distal catheter on the treatment of hydrocephalus: an inflammatory hypothesis? Experience with six cases. Childs Nerv Syst 2006;22(10):1225-30. 